\begin{tabular}{|c|l|}
\hline Title & Coil-globule transition of poly(methyl methacrylate) in isoamyl acetate \\
\hline Author(s) & Nakata, Mitsuo; Nakagawa, Tomohide \\
\hline Citation & $\begin{array}{l}\text { PHY SICAL REVIEW E, 56/3), 3338.3345 } \\
\text { https://doi.org/L0.1103/PhySRevE.56.3338 }\end{array}$ \\
\hline Issue Date & 1997-09 \\
\hline Doc URL & http://hdl.handle.net/2115/5859 \\
\hline Rights & Copyright $\odot 1997$ A merican Physical Society \\
\hline Type & article \\
\hline File Information & PRE56-3.pdf \\
\hline
\end{tabular}

Instructions for use 


\title{
Coil-globule transition of poly(methyl methacrylate) in isoamyl acetate
}

\author{
Mitsuo Nakata and Tomohide Nakagawa \\ Department of Polymer Science, Faculty of Science, Hokkaido University, Sapporo 060, Japan
}

(Received 18 February 1997)

\begin{abstract}
The coil-globule transition was studied by static light scattering measurements on poly(methyl methacrylate) with the molecular weight $M_{w} \times 10^{-6}=2.35$ and 4.4 in isoamyl acetate. Since the phase separation of the dilute solution occurred very slowly, the measurements could be made in the broad temperature range from near the $\Theta$ temperature $61{ }^{\circ} \mathrm{C}$ to $0{ }^{\circ} \mathrm{C} 30 \mathrm{~min}$ after a quench of the solution. The observed expansion factor $\alpha^{2}$ for the radius of gyration was represented as a function only of $\tau M^{1 / 2}$ and showed a constant value at large $-\tau M^{1 / 2}$ with $\tau$ being $1-\Theta / T$. A quantitative comparison between a recent theory for a contracted coil and the data of $\alpha^{2}$ revealed the coil-globule crossover phenomena. The behavior of plot of $1 / \alpha^{3}$ versus $-\tau M^{1 / 2}$ was distinctly different in the three ranges, i.e., coil range, globule range, and range of a constant $\alpha$. The plot of the observed second virial coefficient $A_{2}$ against temperature yielded a minimum as predicted from a theory of $A_{2}$ below the $\Theta$ temperature. [S1063-651X(97)11809-8]
\end{abstract}

PACS number(s): $36.20 . \mathrm{Ey}, 61.25 . \mathrm{Hq}$

\section{INTRODUCTION}

Since Stockmayer predicted a collapse of a single polymer chain [1], many theoretical investigations have been made on the coil-globule transition of a polymer chain [2-10]. Ptitsyn et al. [2] first formulated the expansion factor $\alpha$ below the theta temperature $\Theta$ by introducing the ternary interaction into the Flory equation [11] as

$$
\alpha^{5}-\alpha^{3}-C_{f} / \alpha^{3}=B_{f} \tau M^{1 / 2},
$$

where $\tau$ is defined by $1-\Theta / T, M$ is the molecular weight, and $B_{f}$ and $C_{f}$ are constants associated with the second and third virial coefficients for segment interactions, respectively. Recently, another type of $\alpha$ equation was derived for a contracted chain as [9]

$$
\alpha^{3}-\alpha-C\left(\alpha^{-3}-1\right)=B \tau M^{1 / 2},
$$

where $B$ and $C$ are constants with the same meaning as $B_{f}$ and $C_{f}$ in Eq. (1), respectively.

Both Eqs. (1) and (2) predict the two characteristic features for the coil-globule transition: the transition may occur continuously or discontinuously depending on the magnitudes of the ternary interaction. At the globule state the segment density in a polymer domain becomes independent of the molecular weight. Experimental studies of the coilglobule transition have been made for solutions of polystyrene [12-18] and poly( $N$-isopropylacrylamide) (PNIPAM) [19-21]. For polystyrene solutions a collapse of the chain was not found in the stable state $[22,23]$ but was observed below the phase separation temperature at extreme conditions of low concentration or high solvent viscosity $[14,17,24,25]$. For PNIPAM solutions a collapse of the chain has been measured near and/or below the phase separation temperature but was not analyzed relevantly [20,21]. The above experimental studies are not reliable enough to reveal the globule state predicted by Eqs. (1) and (2), and the discontinuous transition has not been observed for synthetic polymers.
In a previous study [26], dilute solutions of poly(methyl methacrylate) (PMMA) in the mixed solvent tert-butyl alcohol (TBA) + water were found to give rise to very slow phase separation and to remain transparent for a long time after a quench below the cloud point temperature. Taking advantage of this nature we made light scattering measurements for PMMA with the molecular weight $M_{w}=2.38 \times 10^{6}$ and determined the molecular weight $M$, radius of gyration $\left\langle s^{2}\right\rangle$, and second virial coefficient $A_{2}$ in a wide temperature range below the theta temperature $\Theta$. The observed expansion factor $\alpha^{2}=\left\langle s^{2}\right\rangle /\left\langle s^{2}\right\rangle_{0}$ was compared quantitatively with Eq. (2) but only qualitatively with Eq. (1). A collapse of the PMMA chain and a crossover point between the coil and globule ranges were elucidated by Eq. (2).

TBA as well as water cannot dissolve PMMA, but the mixture of these liquids obtains a strong solvent power for the polymer [27]. Though the mixture TBA+water has been speculated to have a specific local structure or clustering of the molecules, we are not certain whether the observed slow phase separation and the collapse of the PMMA chain are attributed to the nature of the polymer or to the properties of the mixture TBA+water. Thus, it is important to investigate a collapse of PMMA chain in a single solvent. A comparison of the collapsed chains in the mixed solvent and a single solvent may reveal universal and specific properties of the coil-globule transition of the polymer chain.

In this study, we found out a very slow phase separation for dilute solutions of PMMA in isoamyl acetate (IAA) and carried out static light-scattering measurements below the cloud-point temperature for two PMMA samples with different molecular weights. The light-scattering measurements, which were made $30 \mathrm{~min}$ after a quench, yielded the correct molecular weights and reliable values of $\left\langle s^{2}\right\rangle$ in a wide temperature range from the $\Theta$ temperature to far below the cloud-point temperature. Based on the plots due to Eq. (2) and $\alpha^{-3}$ versus $-\tau M^{1 / 2}$ the present data of $\alpha^{2}$ in IAA were compared quantitatively with those in the mixed solvent TBA+ water. 


\section{EXPERIMENT AND DATA ANALYSES}

In a previous study PMMA was prepared by bulk polymerization of the monomer and separated into 16 fractions by the fractional solution method [26]. For the present lightscattering measurements we employed the eighth and the thirteenth fractions from this fraction series. The eighth fraction $F 8$ is the same one used in the previous study and the weight average molecular weight was determined to be $M_{w}$ $=2.35 \times 10^{6}$ with IAA as a solvent. The thirteenth fraction $F 13$ has a larger molecular weight $M_{w}=4.4 \times 10^{6}$ as expected from the fractionation method. For measurements of refractive index increment we used a low molecular weight sample with $M_{w}=3.95 \times 10^{4}$, which was fractionated from the original PMMA prepared by bulk polymerization with 2,2-azobis(isobutyronitrile) of $0.20 \mathrm{wt} \%$ and $n$-butyl mercaptane of 1.00 vol \%.

Reagent-grade IAA was washed with saturated sodium carbonate solution and with saturated sodium chloride solution. It was dried with molecular sieves $4 A$ and fractionally distilled in a $1.8 \mathrm{~cm} \times 100 \mathrm{~cm}$ column packed with Raschig rings. The middle fraction at a constant boiling temperature was used for measurements.

The refractive index increment $d n / d c$ for PMMA in IAA was determined with a differential refractometer of the Brice type at the wavelength $436 \mathrm{~nm}$. Since this measurement required relatively high PMMA concentrations up to 0.015 $\mathrm{g} / \mathrm{ml}, d n / d c$ was determined rather in the high temperature range from $38{ }^{\circ} \mathrm{C}$ to $60{ }^{\circ} \mathrm{C}$ to avoid phase separation. $d n / d c$ obtained as a function of temperature $t^{\circ} \mathrm{C}$ was represented by

$$
d n / d c=0.0863+\left(2.7 \times 10^{-4}\right) t
$$

which agreed with values obtained in a previous study [28]. Equation (3) was used to analyze light-scattering data at low temperatures down to $0{ }^{\circ} \mathrm{C}$.

For the light-scattering measurements PMMA solutions were prepared at four concentrations $c(\mathrm{~g} / \mathrm{ml})$ near $c \times 10^{4}$ $=1.2,2.4,3.6$, and 4.8 for the sample $F 8$ and $c \times 10^{4}$ $=0.9,1.8,2.7$, and 3.6 for the sample $F 13$. Each solution was transferred into an optical cell of $18 \mathrm{~mm}$ i.d., sealed with a tightly fitted teflon cap to prevent evaporation of IAA and kept in the dark near the $\Theta$ temperature. Optical clarification of the solutions was made with a Sartorius membrane filter (SM 116, $0.8 \mu \mathrm{m}$ ).

The light-scattering measurements were carried out with unpolarized incident light at $435.8 \mathrm{~nm}$ of a mercury arc as described elsewhere [26,28]. A cylindrical cell filled with the solvent was set at the center of the photometer and kept at a constant temperature within $\pm 0.01 \mathrm{~K}$ by means of a jacket to which thermostatted water was circulated. The optical cell was immersed in the cylindrical cell at a constant temperature and the scattered intensities were measured 30 min after the quench for attainment of thermal equilibrium.

The light-scattering data at an angle $\theta$ were transformed to the excess Rayleigh ratio $R_{\theta}$ and analyzed by the usual scattering equation

$$
\left(K c / R_{\theta}\right)^{1 / 2}=M_{w}^{-1 / 2}+A_{2} M_{w}^{1 / 2} c+(1 / 6)\left\langle s^{2}\right\rangle M_{w}^{-1 / 2} q^{2}
$$

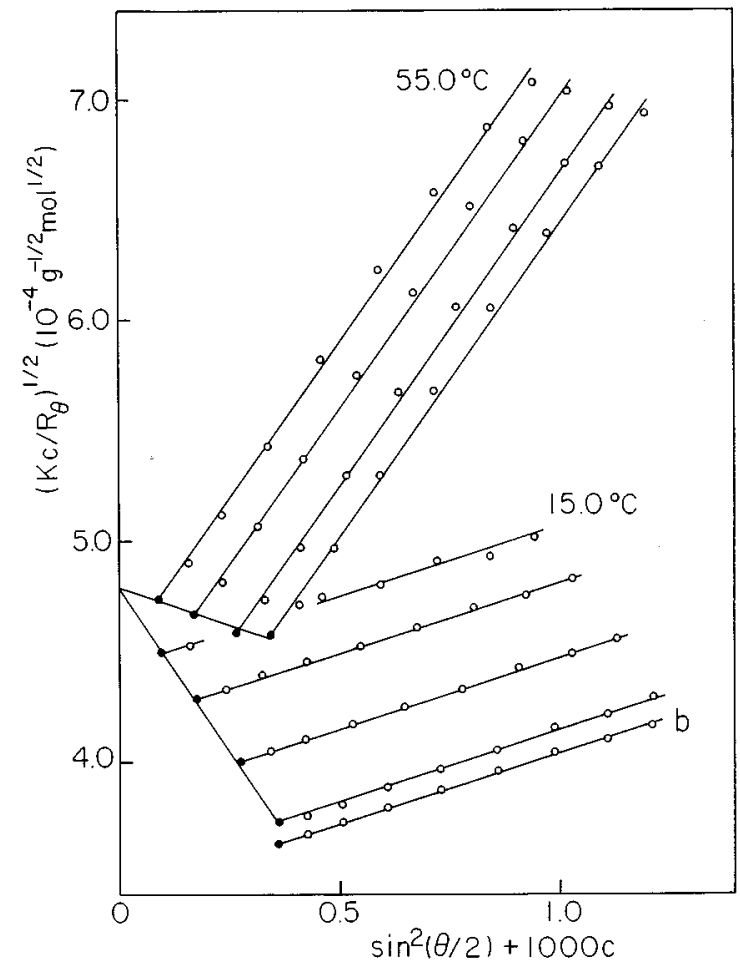

FIG. 1. Light-scattering data by a Zimm plot of $\left(K c / R_{\theta}\right)^{1 / 2}$ as a function of $\sin ^{2}(\theta / 2)$ and $c(\mathrm{~g} / \mathrm{ml})$ according to Eq. (4). The data were obtained for PMMA sample $F 13$ in isoamyl acetate at the indicated temperatures $30 \mathrm{~min}$ after setup of the solution. The plot $b$ was obtained for the solution of the highest concentration at $90 \mathrm{~min}$. The filled circles represent extrapolation to zero angle.

with $K=\left(2 \pi^{2} n^{2} / N_{A} \lambda^{4}\right)(d n / d c)^{2} \quad$ and $\quad q=(4 \pi n /$ $\lambda) \sin (\theta / 2)$, where $N_{A}$ is Avogadro's number, and $\lambda$ is the wavelength of incident light in vacuum.

In Fig. 1 light-scattering data for the sample $F 13$ at $55^{\circ} \mathrm{C}$ and $15^{\circ} \mathrm{C}$ are shown by plotting $\left(K c / R_{\theta}\right)^{1 / 2}$ against $\sin ^{2}(\theta / 2)+1000 c$ in the form of a Zimm plot. The plots of $\left(K c / R_{\theta}\right)^{1 / 2}$ versus $\sin ^{2}(\theta / 2)$ are represented by the straight lines with the same slope and are extrapolated to zero angle as shown by the filled circles. The drastic decrease of the slope caused by the temperature drop from $55^{\circ} \mathrm{C}$ to $15^{\circ} \mathrm{C}$ visualizes a collapse of PMMA coil. $\left(K c / R_{0}\right)^{1 / 2}$ at $\theta=0$ is extrapolated to $c=0$ to determine the molecular weight. The plots at $15^{\circ} \mathrm{C}$ and $55^{\circ} \mathrm{C}$ have the common intercept corresponding to the correct molecular weight. The plot $b$ at $15^{\circ} \mathrm{C}$ shows data for the solution of the highest concentration $90 \mathrm{~min}$ after the quench. This plot indicates a slight increase of scattered intensity but has the same slope as that obtained $30 \mathrm{~min}$ after the quench. The correct common intercept and the small parallel shift of the plot $b$ at $90 \mathrm{~min}$ mean that the very slow phase separation does not disturb the determination of $M$ and $\left\langle s^{2}\right\rangle$.

Figure 2 shows light-scattering data at $38^{\circ} \mathrm{C}$ depicted in the same way as the plots in Fig. 1 . The plots of $\left(K c / R_{\theta}\right)^{1 / 2}$ versus $\sin ^{2}(\theta / 2)$ give the straight lines with a same slope. The extrapolation to $c=0$ of $\left(K c / R_{0}\right)^{1 / 2}$ at $\theta=0$ gives the same intercept as that in Fig. 1. The plots $a$ and $b$ were obtained 60 and $90 \mathrm{~min}$ after the quench, respectively. The relatively large downward shift of the plots indicates considerable increase of scattered intensities with time. The slope 


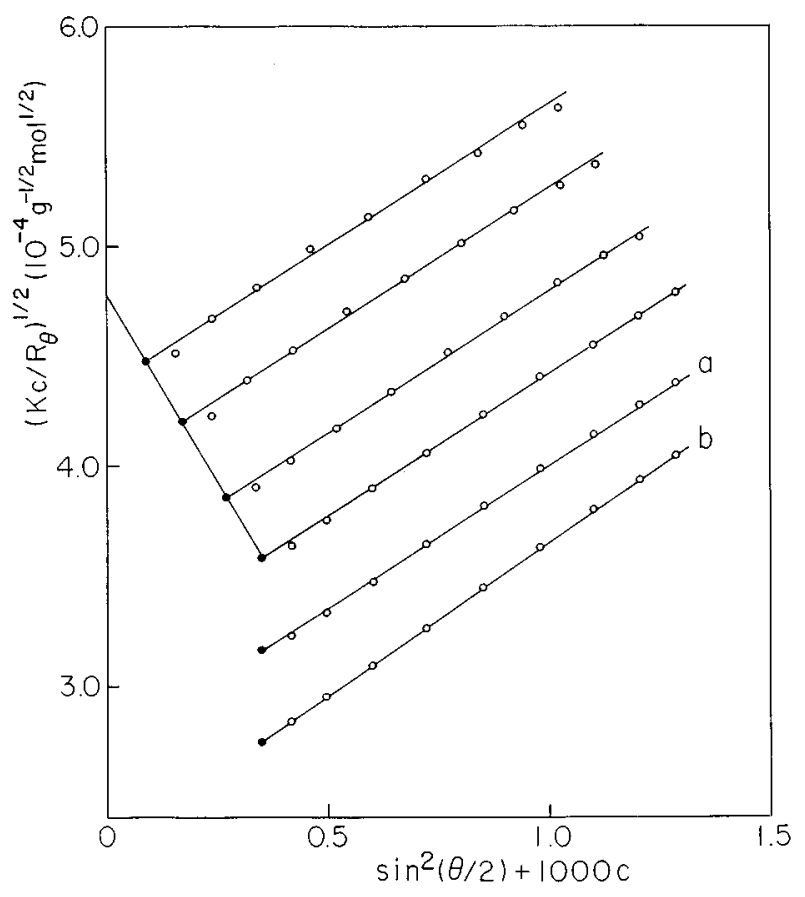

FIG. 2. Light-scattering data by a Zimm plot as in Fig. 1. The data were obtained for PMMA sample $F 13$ in isoamyl acetate at $38.0{ }^{\circ} \mathrm{C} 30 \mathrm{~min}$ after setup of the solution. The plots $a$ and $b$ were obtained for the solution of the highest concentration at 60 and 90 min, respectively. The filled circles represent extrapolation to zero angle.

of the plot $a$ remains unchanged, while the plot $b$ has a slightly larger slope. Again the parallel lines for the plots of angular dependence at 30 and $60 \mathrm{~min}$ and the intercept yielding the correct molecular weight could justify the evaluation of $\left\langle s^{2}\right\rangle$. For the solutions of the highest concentration the phase separation was observed roughly below $45^{\circ} \mathrm{C}$ for the sample $F 8$ and $48^{\circ} \mathrm{C}$ for $F 13$. In Figs. 1 and 2 it is noticeable that the phase separation occurs faster at $38{ }^{\circ} \mathrm{C}$ than at $15^{\circ} \mathrm{C}$. The same trend was also observed in a previous study [26].

The second virial coefficient $A_{2}$ estimated from the line at $\theta=0$ is obviously affected by the effect of the phase separation. At the highest concentration the value of $\left(K c / R_{0}\right)^{1 / 2}$ at zero time could be inferred from the data at 30,60 , and 90 $\min$. The slope of the line at $\theta=0$ was corrected with the value of $\left(K c / R_{0}\right)^{1 / 2}$ at $0 \mathrm{~min}$ and used for a rough evaluation of $A_{2}$. Thus, we evaluated $M,\left\langle s^{2}\right\rangle$, and $A_{2}$ from plots as in Figs. 1 and 2 and listed the values of $\left\langle s^{2}\right\rangle$ in Table I.

In Figs. 3 and $4,\left\langle s^{2}\right\rangle / M$ and $A_{2}$ are plotted against temperature, respectively. The filled and open circles indicate the present data for the samples $F 8$ and $F 13$, respectively. In Fig. 4 the triangles show previous data for $F 8$ in the mixed solvent TBA+water [26]. The solid lines in Figs. 3 and 4 depict the behavior of the data points. To analyze the data for $\left\langle s^{2}\right\rangle$ and $A_{2}$, it is necessary to determine the $\Theta$ temperature and the unperturbed dimension $\left\langle s^{2}\right\rangle_{0}$. In Fig. 4 the increase of $A_{2}$ with increasing temperature seems to level off near $60{ }^{\circ} \mathrm{C}$. Since the present measurements are not suitable for reliable determination of $A_{2}$ because of low polymer concentration, we carried out measurements on a sample of lower molecular weight at higher concentrations. This measure-
TABLE I. Mean square radius of gyration $\left\langle s^{2}\right\rangle$ obtained for PMMA samples $F 8$ with $M_{w}=2.35 \times 10^{6}$ and $F 13$ with $M_{w}=4.4$ $\times 10^{6}$ in isoamyl acetate at various temperatures.

\begin{tabular}{lccc}
\hline \hline $\begin{array}{l}\text { Temp. } \\
\left({ }^{\circ} \mathrm{C}\right)\end{array}$ & $\begin{array}{c}\left\langle s^{2}\right\rangle \\
\left(10^{-11} \mathrm{~cm}^{2}\right)\end{array}$ & $\begin{array}{c}\text { Temp. } \\
\left({ }^{\circ} \mathrm{C}\right)\end{array}$ & $\begin{array}{c}\left\langle s^{2}\right\rangle \\
\left(10^{-11} \mathrm{~cm}^{2}\right)\end{array}$ \\
\hline \multicolumn{4}{c}{ Sample $F 8$} \\
68 & 1.61 & 33 & 0.62 \\
65 & 1.48 & 30 & 0.54 \\
63 & 1.49 & 28 & 0.46 \\
61 & 1.42 & 25 & 0.43 \\
58 & 1.38 & 23 & 0.40 \\
55 & 1.23 & 20 & 0.34 \\
53 & 1.21 & 18 & 0.35 \\
50 & 1.15 & 15 & 0.32 \\
48 & 1.04 & 13 & 0.32 \\
45 & 0.97 & 10 & 0.27 \\
43 & 0.86 & 8 & 0.28 \\
40 & 0.79 & 5 & 0.27 \\
37 & 0.69 & 3 & 0.26 \\
35 & 0.68 & 0 & 0.26
\end{tabular}

\begin{tabular}{llll}
65 & 2.87 & 30 & 0.68 \\
63 & 2.83 & 28 & 0.60 \\
60 & 2.52 & 25 & 0.56 \\
58 & 2.46 & 23 & 0.54 \\
55 & 2.40 & 20 & 0.47 \\
53 & 2.10 & 18 & 0.51 \\
50 & 1.98 & 15 & 0.48 \\
48 & 1.72 & 13 & 0.48 \\
45 & 1.57 & 10 & 0.50 \\
43 & 1.37 & 8 & 0.50 \\
40 & 1.09 & 6 & 0.50 \\
38 & 0.99 & 5 & 0.52 \\
35 & 0.83 & 3 & 0.49 \\
33 & 0.76 & 0 & 0.48 \\
\hline \hline
\end{tabular}

ment showed that with increasing temperature $A_{2}$ increased rapidly for $A_{2}<0$ and slowly near $A_{2}=0$ indicating $61^{\circ} \mathrm{C}$ as a $\Theta$ temperature. $\left\langle s^{2}\right\rangle_{0} / M$ at the $\Theta$ temperature may be independent of molecular weight and insensitive to solvent nature. In Fig. 3 the plots of $\left\langle s^{2}\right\rangle / M$ versus temperature for the samples $F 8$ and $F 13$ appear to cross each other near $61^{\circ} \mathrm{C}$, where the ordinate gives $\left\langle s^{2}\right\rangle / M=6.1 \times 10^{-18} \mathrm{~cm}^{2}$. Moreover, this ratio agrees with those determined at the $\Theta$ temperature in the mixture TBA+water [26] and in 1-chlorobutane [28]. Thus, we can determine the $\Theta$ condition as $\Theta=61.0^{\circ} \mathrm{C}$ and $\left\langle s^{2}\right\rangle_{0} / M=6.1 \times 10^{-18} \mathrm{~cm}^{2}$ for the present system.

\section{COIL-GLOBULE TRANSITION BEHAVIOR}

Figure 5 gives a plot of $\alpha^{2}\left(=\left\langle s^{2}\right\rangle /\left\langle s^{2}\right\rangle_{0}\right)$ against $-\tau M^{1 / 2}$. The present data for samples $F 8$ and $F 13$ are given by the filled and open circles, respectively. The triangles are for the previous data for $F 8$ in TBA+water [26]. As expected from Eqs. (1) and (2) the present data points of the two different molecular weights give a composite curve in- 


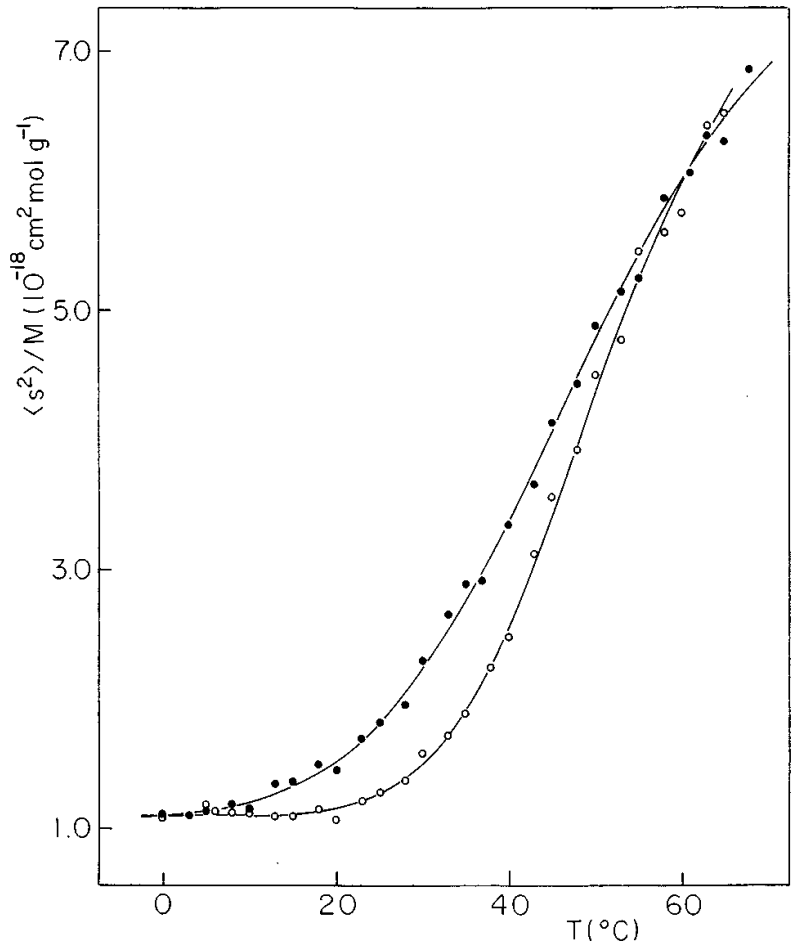

FIG. 3. Ratio of mean square radius of gyration to molecular weight $\left\langle s^{2}\right\rangle / M$ as a function of temperature $T$ for PMMA in isoamyl acetate. The filled and open circles are for samples $F 8$ and $F 13$, respectively. The solid lines are guides to the eye.

dependent of $M$. For the system of TBA+water $\alpha^{2}$ decreases rapidly with increasing $-\tau M^{1 / 2}$ and becomes 0.11 near $-\tau M^{1 / 2}=100$, while for the system of IAA $\alpha^{2}$ decreases relatively slowly and levels off with a value near

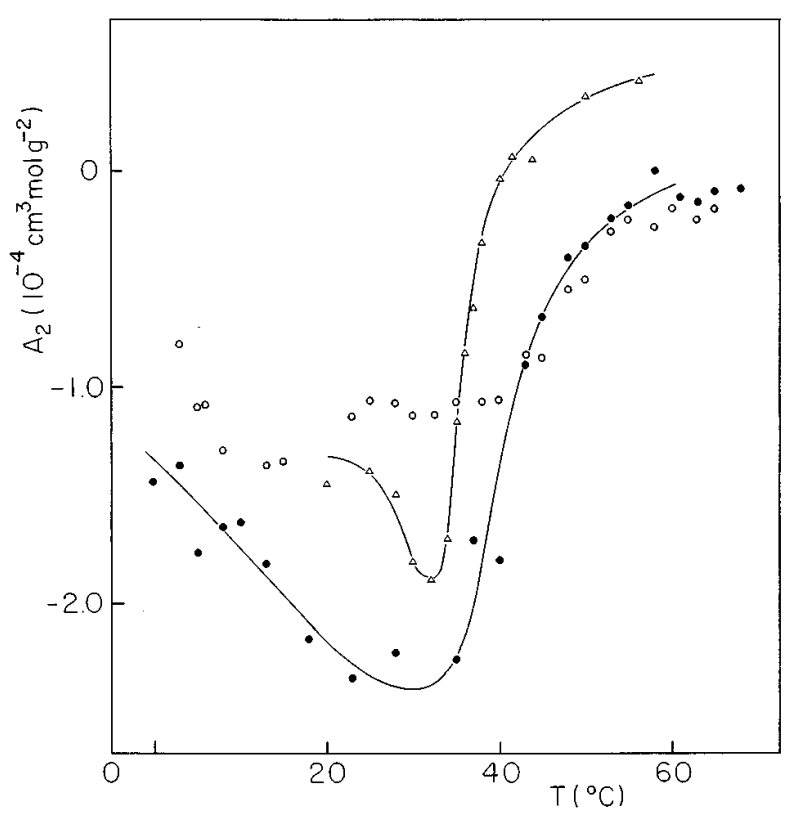

FIG. 4. Plot of second virial coefficient $A_{2}$ versus temperature $T$. The filled and open circles are for PMMA samples $F 8$ and $F 13$ in isoamyl acetate, respectively. The triangles are for $F 8$ in tertbutyl alcohol+water (Ref. [26]).

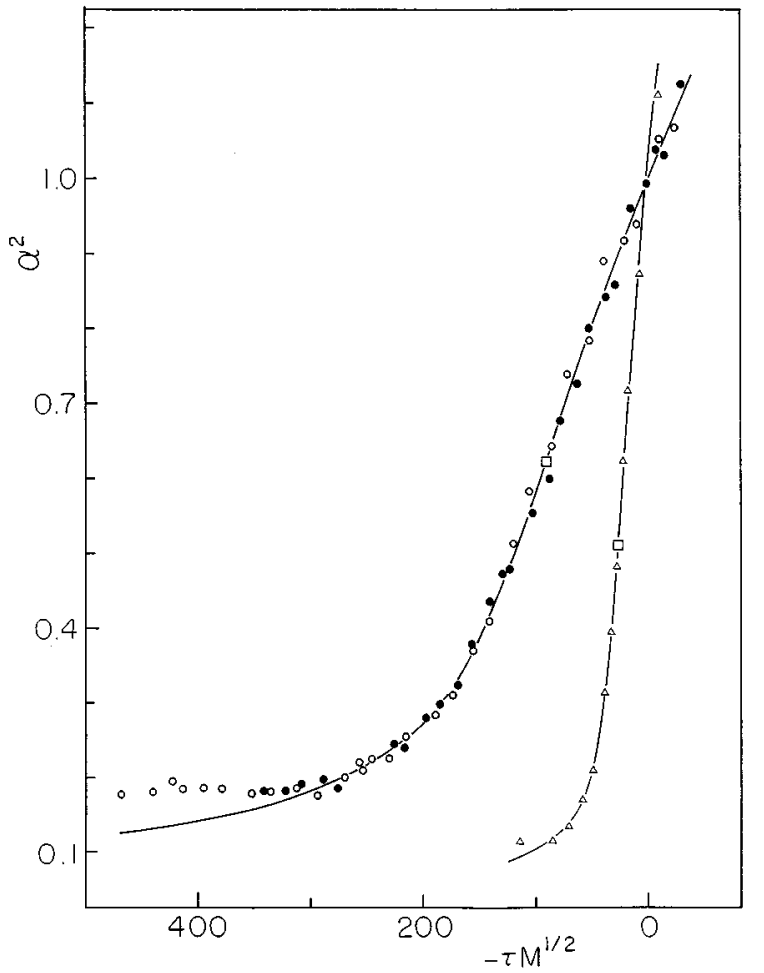

FIG. 5. Plot of expansion factor $\alpha^{2}$ vs $-\tau M^{1 / 2}$ with $\tau=1$ $-\Theta / T$ and molecular weight $M(\mathrm{~g} / \mathrm{mol})$. The filled and open circles are for PMMA samples $F 8$ and $F 13$ in isoamyl acetate, respectively. The triangles are for $F 8$ in tert-butyl alcohol+water (Ref. [26]). The solid lines are described by Eq. (2). The squares on the lines represent a crossover point between coil and globule ranges.

0.18 for $-\tau M^{1 / 2}>300$. Equations (1) and (2) can be compared with the experimental data, respectively, by the plots of $\alpha^{8}-\alpha^{6}$ versus $\alpha^{3} \tau M^{1 / 2}$ and $\left(\alpha^{3}-\alpha\right) /\left(1-\alpha^{-3}\right)$ versus $\tau M^{1 / 2} /\left(1-\alpha^{-3}\right)$. The plot of $\alpha^{8}-\alpha^{6}$ versus $\alpha^{3} \tau M^{1 / 2}$ was largely curved and a quantitative agreement between Eq. (1) and the experimental data was not obtained. Figure 6 shows the plot due to Eq. (2). Data near the $\Theta$ temperature, i.e., $\left(\alpha^{3}-\alpha\right) /\left(1-\alpha^{-3}\right)=2 / 3$, are not given on account of large uncertainty caused by a small error in $\alpha$. The data points for $-\tau M^{1 / 2}>300$ in Fig. 5 are located horizontally near $\left(\alpha^{3}\right.$ $-\alpha) /\left(1-\alpha^{-3}\right)=0.03$, above which the data points for both the samples may be represented by the straight line, from which we estimated $B=0.0041$ and $C=0.073$. In Fig. 5 the solid line for the system of IAA is calculated by Eq. (2) with these values of $B$ and $C$. The solid line for the system of TBA+ water is described by Eq. (2) with $B=0.0160$ and $C$ $=0.044$ obtained in the previous study.

In Fig. 7 the data for the systems of IAA and TBA+ water are given by the plot of $-\alpha^{3} \tau M^{1 / 2}$ versus $-\tau M^{1 / 2}$ with the same symbols as in Fig. 5. The two plots for the different systems are similar in shape but largely different in scale. The solid lines for the two systems are described by Eq. (2) with the above values of $B$ and $C$. The horizontal lines at larger $-\tau M^{1 / 2}$ indicate the asymptotic globule state due to Eq. (2), i.e., $-\alpha^{3} \tau M^{1 / 2}=C / B=17.8$ for the system of IAA and 2.75 for the system of TBA+water. In the experimental ranges the solid lines approach the respective horizontal 


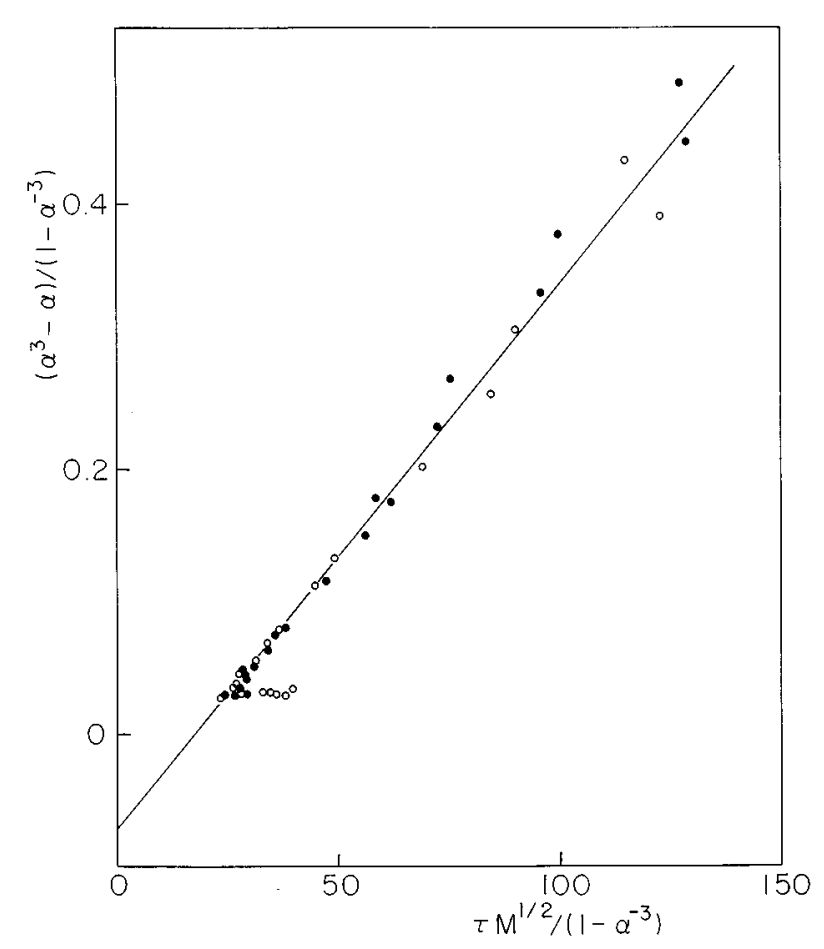

FIG. 6. Plot according to Eq. (2) for PMMA samples $F 8$ and $F 13$ in isoamyl acetate. Symbols are the same as in Fig. 5. The slope and intercept of the straight line give $B=0.0041$ and $C=0.073$ for the constants in Eq. (2), respectively.

lines, while the data points especially for the system of IAA deviate upward before reaching the globule state. This upward deviation corresponds to the constant value of $\alpha^{2}$ indicated in Fig. 5. In the previous study [26] we explained the upward deviation as due to the effect of the higher order interactions beyond the third virial coefficient.

The segment volume fraction $\phi$ in a polymer domain may be given by

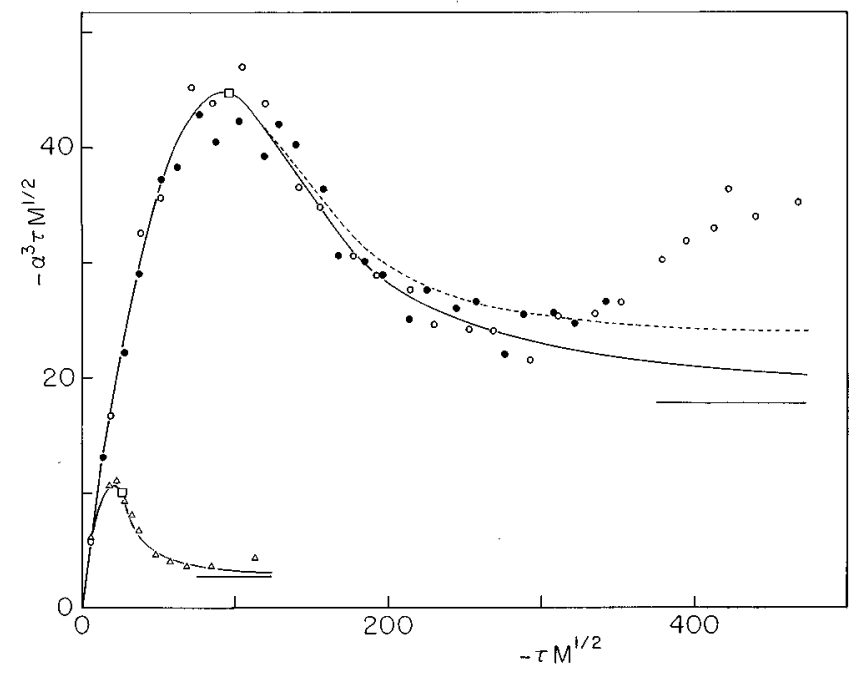

FIG. 7. Plot of $-\alpha^{3} \tau M^{1 / 2}$ vs $-\tau M^{1 / 2}$ for PMMA samples $F 8$ and $F$ 13. Symbols are the same as in Fig. 5. The solid lines are due to Eq. (2) and the horizontal lines indicate the asymptotic limit $C / B$. The dotted line is due to Eqs. (2) and (6). The squares near the maximum are crossover point.

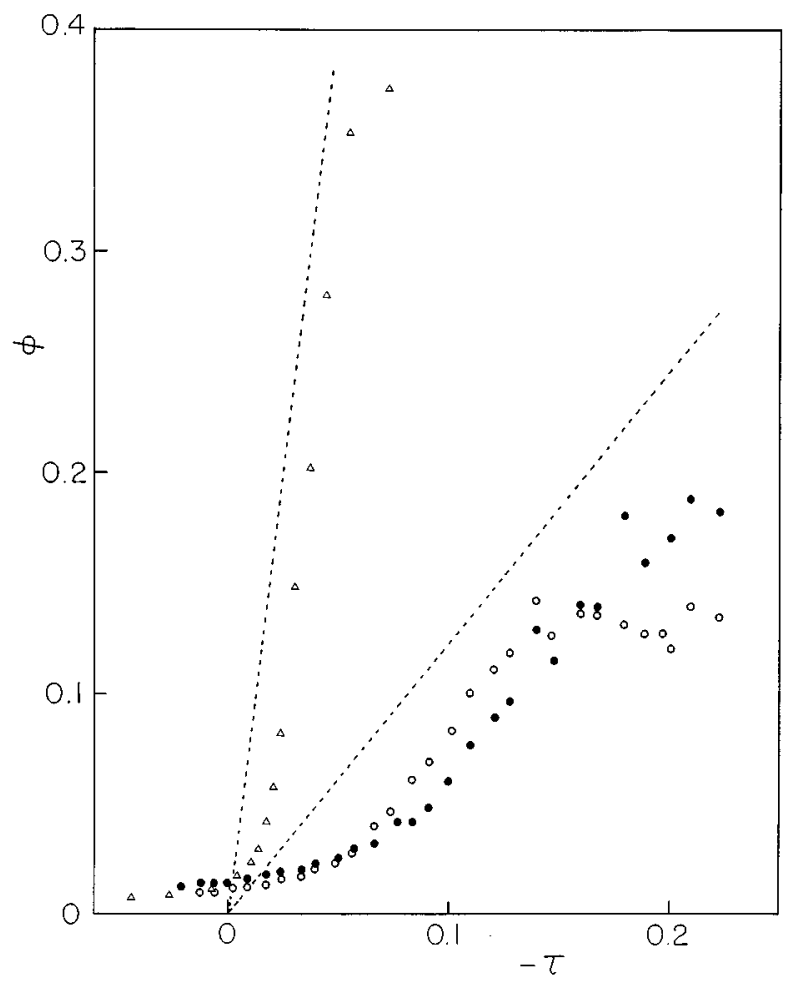

FIG. 8. Segment volume fraction $\phi$ vs reduced temperature $-\tau$ for PMMA samples $F 8$ and $F 13$. Symbols are the same as in Fig. 5. The dotted lines represent asymptotic relation due to Eq. (2).

$$
\phi=(M / \rho) /\left\{(4 \pi / 3) \kappa\left\langle s^{2}\right\rangle^{3 / 2} N_{A}\right\}
$$

where $\rho$ is the density of PMMA in the liquid state and $\kappa$ may be unity for a coil state and $(5 / 3)^{3 / 2}$ for a globule state. $\phi$ was evaluated for the two systems by using $\rho=1.20 \mathrm{~g} / \mathrm{ml}$ at $25{ }^{\circ} \mathrm{C}$ and $\kappa=1$. Figure 8 shows a plot of $\phi$ versus $-\tau$ with the same symbols as in Fig. 5. The asymptotic relation due to Eq. (2) yields $\phi=-1.23 \tau$ for the system of IAA and $\phi=-8.0 \tau$ for the system of TBA+water, which are indicated by the dotted lines. For the system of IAA, $\phi$ increases similarly for the two samples with increasing $-\tau$ for $-\tau$ $<0.15$ but behaves differently for $-\tau>0.15$ contrary to the asymptotic relation $\phi=-1.23 \tau$. For the system of TBA + water $\phi$ increases rapidly with increasing $-\tau$ and attains a high value such as 0.38 , which is twice as large as the maximum value for the sample $F 8$ in IAA. For the sample $F 13$ in IAA the maximum of $\phi$ remains near 0.14 . The value of $\phi$ will reduce to half when we employ $\kappa=(5 / 3)^{3 / 2}$. In any case the segment density in the polymer domain is not dilute in the usual sense. The effect of higher order interactions could be expected to cause a large deviation of data points from Eq. (2) for the system of TBA+water because of the large $\phi$. Though this is not the case as shown in Fig. 7, we estimated the effect of the higher order interactions beyond the ternary interaction on Eq. (2).

Sanchez derived an equation of $\alpha$ in which all higher order interactions are approximately taken into account [6]. The equation may be obtained by replacing the constant $C_{f}$ by $C_{f} Y(\phi)$ in Eq. (1), where $Y(\phi)$ is given by

$$
Y(\phi)=-3\left\{\ln (1-\phi)+\phi+(1 / 2) \phi^{2}\right\} / \phi^{3}
$$


and $\phi$ is by Eq. (5) with $\kappa=1$. In the Sanchez theory $\alpha$ becomes dependent on $M$ as well as on $\tau M^{1 / 2}$ through the relation $\phi \sim M^{-1 / 2} \alpha^{-3}$. As in the case of Eq. (1) the higher order interactions may be also introduced in Eq. (2) by using $C Y(\phi)$ instead of $C$.

In Fig. 7 the dotted line, which agrees with the solid line for $-\tau M^{1 / 2}<120$, is evaluated for the sample $F 13$ by Eqs. (2) and (6). In the flat region for large $-\tau M^{1 / 2}$ the broken line has a very shallow minimum of about 24 around 470 . For the sample $F 8$ Eqs. (2) and (6) give a similar line with a minimum of 25 near $-\tau M^{1 / 2}=420$. We replaced $C$ in Eq. (2) by $C\left\{1+D /\left(M^{1 / 2} \alpha^{3}\right)\right\}$ to estimate the effect of the quaternary interaction. The modified Eq. (2) with $D=20$ gave lines numerically close to those due to the Sanchez theory. The use of larger $D$ yielded lines with a shallow minimum of a higher value at smaller $-\tau M^{1 / 2}$. Thus, the marked upward deviation of data points from Eq. (2) cannot be explained reasonably by the effect of the higher order interactions.

It is relevant to clarify whether the terms of $B$ and $C$ in Eq. (2) may be related quantitatively to the second virial coefficient $v \tau$ and the third virial coefficient $w$ for segment interaction, respectively, or should be taken as phenomenological parameters. The straight line fit in Fig. 6 validates the functional form of Eq. (2) but cannot answer to this question. Equation (2) can be explicitly expressed with $v \tau$ and $w$ by using $(5 / 3)^{3 / 2}(4 \pi / 3)\left\langle s^{2}\right\rangle^{3 / 2}$ for the volume occupied by a polymer chain $[9,10]$. This formulation gives $B$ and $C$ in Eq. (2) as

$$
\begin{gathered}
B=1.09 v(N / M)^{1 / 2} / a^{3}, \\
C=3.55 w / a^{6},
\end{gathered}
$$

where $a$ is the segment size and $N$ is the segment number per chain. The relation $\left\langle s^{2}\right\rangle_{0}=a^{2} N / 6$ and the molecular weight $m=M / N$ per segment transform $B$ and $C$ as $B$ $=0.0740 v /\left(\left\langle s^{2}\right\rangle_{0} / M\right)^{3 / 2} m^{2}$ and $C=0.0164 w(M / m)^{3} /\left\langle s^{2}\right\rangle_{0}^{3}$. Thus, $v$ and $w$ for the monomer unit $m=100$ of PMMA can be evaluated from the values of $B$ and $C$, respectively. We obtained $v=0.84 \times 10^{-23} \mathrm{~cm}^{3}$ and $w=1.01 \times 10^{-45} \mathrm{~cm}^{6}$ for the system of IAA and $v=3.3 \times 10^{-23} \mathrm{~cm}^{3}$ and $w=0.61$ $\times 10^{-45} \mathrm{~cm}^{6}$ for the system of TBA+ water. The chain stiffness parameter $w^{1 / 2} / a^{3}[9,10]$ is calculated as 0.143 and 0.111 for the systems of IAA and TBA+ water, respectively.

In recent light-scattering studies the ternary cluster integral $\beta_{3}(=3 w)$ per monomer unit has been estimated as 4.8 $\times 10^{-45} \mathrm{~cm}^{6}$ for PMMA in acetonitrile and $4.4 \times 10^{-45} \mathrm{~cm}^{6}$ for polystyrene in cyclohexane at the $\Theta$ temperature [29]. It is remarkable that the above values of $w$ due to $C$ are of the same order of magnitude as those obtained by the lightscattering measurement. For PMMA in IAA the temperature dependence of the second virial coefficient $A_{2}$ below the $\Theta$ temperature has been measured for low molecular weight samples [28,30]. Assuming the relation $A_{2}=\left(N_{A} / 2 m^{2}\right) 2 v \tau$ [1] we obtained $v=1.09 \times 10^{-23} \mathrm{~cm}^{3}$ per monomer unit, which is close to the above value estimated from $B$. The reasonable agreement between the virial coefficients due to Eq. (2) and that due to the other experiments may justify Eq. (2). Consequently, the failure of the introduction of the higher order interactions in Eq. (2) suggests another collapse mechanism for the deviation of the data points from Eq. (2)

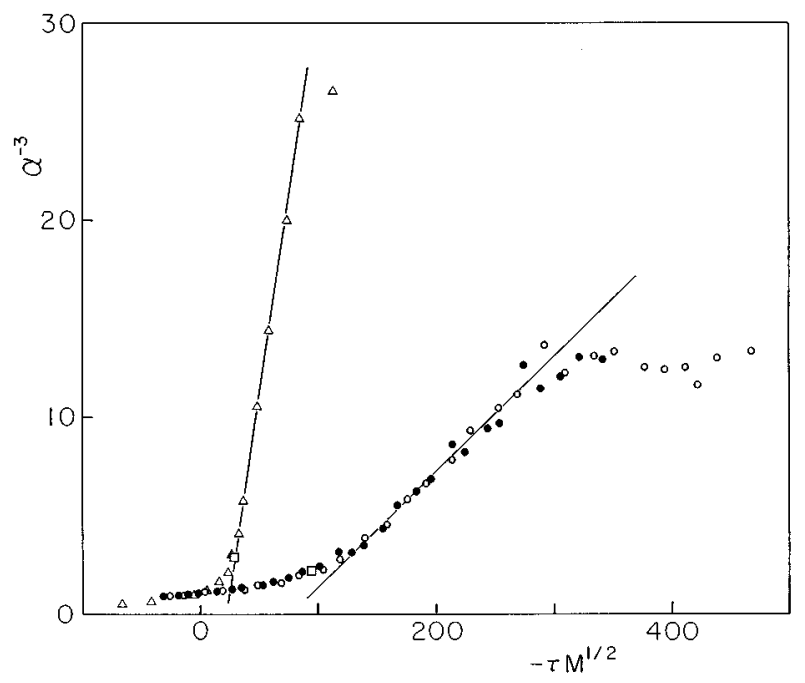

FIG. 9. Plot of $1 / \alpha^{3}$ vs $-\tau M^{1 / 2}$ for PMMA samples $F 8$ and $F 13$. Symbols are the same as in Fig. 5. The straight lines fitted to data points indicate a globule range according to Eq. (9) and (10). The squares are the crossover point.

at large $-\tau M^{1 / 2}$. Thus, the collapse of the PMMA chain can be considered to occur through the three stages, the coil region, globule region, and the region characterized by a constant $\alpha$. The transition to the last state occurs abruptly as shown in Figs. 6 and 7, while the crossover point between the coil and globule regions is obscured in the smooth lines in Figs. 5-7.

According to Eq. (2) the coil-globule transition occurs continuously or discontinuously depending on the value of $C$. The critical conditions $\left(d \alpha^{k} / d x\right)^{-1}=\left(d^{2} \alpha^{k} / d x^{2}\right)^{-1}=0$ give the critical values $C=0.00549$ and $\alpha^{2}=0.471$, where $k$ is an integer and $x=B \tau M^{1 / 2}$. Correspondingly, $\left(d^{2} \alpha^{k} / d x^{2}\right)^{-1}=0$ may be used as a condition for the crossover point and yields the crossover value $\alpha^{2}=0.53(k=1)$, $0.62(2)$, and $0.81(3)$ for the system of IAA and $0.45(k$ $=1), 0.51(2)$, and $0.63(3)$ for the system of TBA+water. Though the reasonable value of $k$ is not certain, the condition indicates a relatively wide range for the globule state.

For the behavior of $\alpha$ in the globule range Grosberg presented the following relation [10]:

$$
\alpha=\left(w / a^{6}\right)^{1 / 4} \widetilde{\alpha}(t),
$$

where the argument $t$ was defined by $t$ $=6^{3 / 4} v \tau N^{1 / 2} /\left(w a^{6}\right)^{1 / 4}$ and the dimensionless function $\widetilde{\alpha}(t)$ was calculated numerically. We found that $\widetilde{\alpha}(t)$ is well approximated by

$$
\widetilde{\alpha}^{-3}(t)=-0.70-0.086 t .
$$

Equations (9) and (10) indicate that the plot of $\alpha^{-3}$ versus $\tau M^{1 / 2}$ should yield a straight line, from which $v$ and $w$ can be estimated. In Fig. $9 \alpha^{-3}$ is plotted against $-\tau M^{1 / 2}$ with the same symbols as in Fig. 5. The plot for the system of IAA can be divided distinctly into three parts. As expected from Eqs. (9) and (10) the data points in the middle range are well represented by the straight line, from which we obtained $v=3.3 \times 10^{-23} \mathrm{~cm}^{3}$ and $w^{1 / 2} / a^{3}=0.28$. The plot for the system of TBA+water consists of two parts of different slope. 
The straight line was fitted to the points in the globule range and $v=9.0 \times 10^{-23} \mathrm{~cm}^{3}$ and $w^{1 / 2} / a^{3}=0.18$ were obtained. Grosberg located the coil-globule transition point at $t=$ -10.2 , which may provide a measure of the crossover point for a system of large $w^{1 / 2} / a^{3}$. By using the values of $v$ and $w^{1 / 2} / a^{3}$ obtained above, we estimated the crossover point as $\tau M^{1 / 2}=-97$ and $\alpha^{2}=0.60$ for the system of IAA, and $\tau M^{1 / 2}=-28$ and $\alpha^{2}=0.49$ for the system of TBA+water. In Fig. 9 these crossover points are noticeable as a point where data points just approach respective straight lines from the left. Moreover these crossover points are very close to those obtained by the condition $\left(d^{2} \alpha^{2} / d x^{2}\right)^{-1}=0$. Thus, the crossover point due to this condition $(k=2)$ is given by the square in Figs. 5, 7, and 9. The values of $v$ and $w^{1 / 2} / a^{3}$ determined by Eqs. (9) and (10) are consistently a few times larger than those estimated by Eq. (2). This difference cannot be taken seriously on account of different assumptions in the derivation of Eqs. (2) and (9). It should be noticed that the plot of $\alpha^{-3}$ versus $-\tau M^{1 / 2}$ is very sensitive to the crossover between the coil and globule regions.

Recently, Szleifer et al. [31] and Tanaka et al. [32] performed Monte Carlo simulations in the ranges of chain length from 20 to 100 and from 40 to 1000, respectively, and demonstrated that $\alpha^{3}$ is a function only of $\tau N^{1 / 2}$ below the $\Theta$ temperature. Each plot of $-\alpha^{3} \tau N^{1 / 2}$ versus $-\tau N^{1 / 2}$ obtained by these simulations exhibited a curve with a maximum similar to those by Eq. (2). In the flat region after the maximum, data points by Szleifer showed a slight increase with increasing $-\tau N^{1 / 2}$. This was explained by the fact that the chain had a constant $\alpha$ at a completely collapsed state and the increase of $-\alpha^{3} \tau N^{1 / 2}$ was caused by the increase of $-\tau N^{1 / 2}$. In Fig. 7 the upward deviation of the data points for the system IAA cannot be attributed to this effect, because as shown in Fig. 5 PMMA chains in IAA at large $-\tau M^{1 / 2}$ have evidently a larger size than those in TBA+water and are not completely collapsed. In the simulation by Tanaka et al. the upward deviation is not observed in the flat region where the segment density is expected to be extremely high.

In Fig. 4 the plot of $A_{2}$ versus temperature exhibits a pronounced minimum for the sample $F 8$ in both the solvents. The irregular behavior of the data points for $F 13$ in IAA may be mainly ascribed to experimental uncertainty, because the data were determined at low concentrations. Tanaka [33] considered experimental data of $A_{2}$ below the $\Theta$ temperature [12] and derived an equation of $A_{2}$ taking into account the ternary interaction and polymer size through Eq.
(1). This theoretical calculation suggests that $A_{2} N^{1 / 2}$ is a function only of $\tau N^{1 / 2}$ and $A_{2}$ has a marked minimum at the point where the coil-globule transition occurs. According to the theory we plotted $A_{2} M^{1 / 2}$ against $-\tau M^{1 / 2}$ for the data of the system of IAA. However, we could not observe a well defined composite curve on account of experimental errors. In Fig. 4 the solid lines yield a minimum at $-\tau M^{1 / 2}=160$ for IAA and at -48 for TBA+water. These values of $-\tau M^{1 / 2}$, which remain in the range of the globule state, are not largely different from those given by the condition $\left(d^{2} \alpha^{2} / d x^{2}\right)^{-1}=0$. This reasonable agreement between the theoretical prediction and the experimental data indicates that the study of $A_{2}$ could reveal new aspects of polymer chain in the collapsed state.

In summary, we have studied the coil-globule transition by static light-scattering measurements for two PMMA samples in IAA. The measurements were carried out in a wide temperature range below the $\Theta$ temperature taking advantage of the very slow phase separation of the dilute solutions. The observed $\alpha^{2}$ is a function only of $\tau M^{1 / 2}$ and may be constant at large $-\tau M^{1 / 2}$. The behavior of the plot of $\alpha^{-3}$ versus $-\tau M^{1 / 2}$ is distinctly different in the three ranges of $-\tau M^{1 / 2}$, i.e., the coil range, globule range, and the range of a constant $\alpha$. In both the coil and globule ranges $\alpha$ is compared quantitatively with a theoretical prediction of Eq. (2), and the second and third virial coefficients for segment interaction are estimated reasonably. The plot of $A_{2}$ versus temperature yields a minimum as predicted by a theory of $A_{2}$ below the $\Theta$ temperature. A comparison of the present data with previous ones for the system TBA+ water was made to reveal universal and specific properties of the collapse of PMMA chain. PMMA chain in TBA+water has a more compact form than in IAA but the coil-globule transitions in these solvents give the same behavior described by Eq. (2).

Usually, the reduced temperature $\tau$ is defined by $T / \Theta$ -1 in analyses of chain collapse near the $\Theta$ temperature. The present data were also analyzed with this definition for $\tau$. However, the results were essentially the same as the present ones with $\tau=1-\Theta / T$. The very slow phase separation of dilute solutions of PMMA in IAA is interesting in view of a nucleation process [34] and under investigation.

\section{ACKNOWLEDGMENT}

This work was supported by a Grant-in-Aid for Scientific Research (C, No. 08640496) from the Ministry of Education, Science, Sports, and Culture of Japan.
[1] W. H. Stockmayer, Makromol. Chem. 35, 54 (1960).

[2] O. B. Ptitsyn, A. K. Kron, and Y. Y. Eizner, J. Polym. Sci. Part C 16, 3509 (1968).

[3] P. G. DeGennes, J. Phys. (France) Lett. 36, L55 (1975).

[4] I. M. Lifshitz, A. Yu. Grosberg, and A. R. Khokhlov, Rev. Mod. Phys. 50, 683 (1978).

[5] C. B. Post and B. H. Zimm, Biopolymers 18, 1487 (1979).

[6] I. C. Sanchez, Macromolecules 12, 980 (1979).

[7] E. A. DiMarzio, Macromolecules 17, 969 (1984).

[8] G. Allegra and F. Ganazzoli, J. Chem. Phys. 83, 397 (1985).
[9] T. M. Birshtein and V. A. Pryamitsyn, Macromolecules 24, 1554 (1991).

[10] A. Yu. Grosberg and D. V. Kuznetsov, Macromolecules 25, 1970 (1992); 25, 1980 (1992); 25, 1991 (1992).

[11] P. J. Flory, Principles of Polymer Chemistry (Cornell University Press, Ithaca, 1953).

[12] M. Nierlich, J. P. Cotton, and B. Farnoux, J. Chem. Phys. 69, 1379 (1978).

[13] G. Swislow, S. T. Sun, I. Nishio, and T. Tanaka, Phys. Rev. Lett. 44, 796 (1980). 
[14] S. T. Sun, I. Nishio, G. Swislow, and T. Tanaka, J. Chem. Phys. 73, 5971 (1980).

[15] P. Stepanek, C. Konak, and B. Sedlacek, Macromolecules 15, 1214 (1982).

[16] I. H. Park, Q. W. Wang, and B. Chu, Macromolecules 20, 1965 (1987).

[17] B. Chu, I. H. Park, Q. W. Wang, and C. Wu, Macromolecules 20, 2833 (1987).

[18] J. Yu, Z. L. Wang, and B. Chu, Macromolecules 25, 1618 (1992).

[19] I. Nishio, S. T. Sun, G. Swislow, and T. Tanaka, Nature (London) 281, 208 (1979).

[20] K. Kubota, S. Fujishige, and I. Ando, J. Phys. Chem. 94, 5154 (1990).

[21] C. Wu, and S. Zhou, Macromolecules 28, 8381 (1995).

[22] H. Yamakawa, Macromolecules 26, 5061 (1993).

[23] H. Yamakawa, F. Abe, and Y. Einaga, Macromolecules 27, 5704 (1994).
[24] A. Yu. Grosberg and D. V. Kuznetsov, Macromolecules 25, 1996 (1992).

[25] A. Yu. Grosberg and D. V. Kuznetsov, Macromolecules 26, 4249 (1993).

[26] M. Nakata, Phys. Rev. E 51, 5770 (1995).

[27] J. M. G. Cowie, M. A. Mohsin, and I. J. McEwen, Polymer 28, 1569 (1987).

[28] M. Nakata, K. Kawate, and Y. Ishitaka, Macromolecules 27, 1825 (1994).

[29] H. Yamakawa, F. Abe, and Y. Einaga, Macromolecules 27, 3272 (1994).

[30] R. Kirste and G. V. Schulz, Z. Phys. Chem. Neue Folge 27, 301 (1961).

[31] I. Szleifer, E. M. O'Toole, and A. Z. Panagiotopoulos, J. Chem. Phys. 97, 6802 (1992).

[32] G. Tanaka and W. L. Mattice, Macromol. Theory Simul. 5, 499 (1996).

[33] F. Tanaka, J. Chem. Phys. 82, 4707 (1985).

[34] M. Nakata and K. Kawate, Phys. Rev. Lett. 68, 2176 (1992). 